\title{
Correction to: Regularity Scales and Convergence of the Calabi Flow
}

\author{
Haozhao $\mathrm{Li}^{1} \cdot$ Bing Wang ${ }^{2}$ (D) Kai Zheng ${ }^{3}$ \\ Published online: 26 July 2018 \\ () Mathematica Josephina, Inc. 2018
}

\section{Correction to: J Geom Anal https://doi.org/10.1007/s12220-017-9896-y}

The authors would like to acknowledge additional funding. Corrected acknowledgments appear here.

Acknowledgements The authors would like to thank Professor Xiuxiong Chen, Simon Donaldson, Weiyong He, Claude LeBrun and Song Sun for insightful discussions. Haozhao Li and Kai Zheng would like to express their deepest gratitude to Professor Weiyue Ding for his support, guidance and encouragement during the project. Part of this work was done while Haozhao Li was visiting MIT and he wishes to thank MIT for their generous hospitality. H. Li: Supported by NSFC Grant No. 11671370. B. Wang: Supported by NSF Grant DMS-1312836. K. Zheng: Supported by the EPSRC on a Programme Grant entitled "Singularities of Geometric Partial Differential Equations" Reference Number EP/K00865X/1, and the European Union's Horizon 2020 research and innovation program under the Marie Skłodowska-Curie Grant Agreement No. 703949.

The original article can be found online at https://doi.org/10.1007/s12220-017-9896-y.

$凶 \quad$ Bing Wang

bwang@math.wisc.edu

Haozhao Li

hzli@ustc.edu.cn

Kai Zheng

K.Zheng@warwick.ac.uk

1 Key Laboratory of Wu Wen-Tsun Mathematics, Chinese Academy of Sciences, School of Mathematical Sciences, University of Science and Technology of China, No. 96 Jinzhai Road, Hefei 230026, Anhui, China

2 Department of Mathematics, University of Wisconsin-Madison, Madison, WI 53706, USA

3 Mathematics Institute, University of Warwick, Coventry CV4 7AL, UK 